\title{
Effect of Iron Atoms on the Properties of Silicon Cage Clusters
}

\author{
S. MAhtout* \\ Physics Theory Laboratory, University A/MIRA of Bejaia, 06000, Algeria \\ (Received October 10, 2012; in final form June 11, 2013)
}

\begin{abstract}
In this work, we have systematically studied the effect of iron atoms on the structural, electronic, and magnetic properties of silicon cage clusters in the range size of 19 to 24 atoms, using the density functional theory implemented in the code SIESTA. A new behaviour in the structural, electronic, and magnetic properties of the doped silicon clusters is obtained. We find that the encapsulation of one-Fe atoms within silicon clusters lead to stable Fe encapsulated Si clusters when compared to the clusters with the same size of pure silicon. However, the clusters stabilities leads to a decrease when the number of Fe atoms in substitution increase in the clusters. It is seen that the Fe doped silicon clusters have large HOMO-LUMO gap for spin up electrons while those with spin down electrons have a very small HOMO-LUMO gap. The silicon clusters which are not magnetic in their pure state become magnetic after the substitution of Fe atoms and the magnetic moments of different structures increase when the number of $\mathrm{Fe}$ atoms increase in the clusters.
\end{abstract}

DOI: 10.12693/APhysPolA.124.688

PACS: 61.46.-w, 36.40.Mr, 82.30.Nr

\section{Introduction}

The metal-doped silicon clusters or cages have been the subject of extensive theoretical and experimental investigations [1-11]. The main reason for this is that metal doped silicon cage clusters have found many technological applications and appear to be good candidates for numerous new applications in nanotechnologies. It is well known that silicon does not spontaneously form stable cage structures, since $s p^{2}$ hybridization is highly unfavourable in the case of Si atom [12, 13].

A possible approach to stabilise $\mathrm{Si}$ cage is to locate a transition metal atom in substitution at the surface or in the center of the cluster as suggested by some experimental studies [14] and theoretical calculations [15-19]. These studies have demonstrated that the encapsulation of the transition metal atoms leads to stable silicon cages. Encapsulation of transition metal $(\mathrm{Fe})$ elements in the cage of silicon clusters exhibits many novel behaviours, because the transition metal atoms can saturate the dangling bond on the silicon cage surface [17, 20].

Charge transfer, magnetism and other properties of the metal encapsulated silicon clusters leads to different properties for doped clusters when compared to pure silicon clusters. In cluster research, structural stability, electronic structure, and magnetic properties are three important interdependent parameters, since the magnetic moment depends on the electronic structure, which in turn depends on the geometry. Knowledge concerning this dependence can give us additional information to the physical and chemical properties of the clusters. Hiura

*e-mail: mahtout_sofiane@yahoo.fr et al. [14] have shown that the $\mathrm{Si}_{12} \mathrm{~W}$ is a very stable cluster, and its geometry is that of two hexagons of Si atoms sandwiching a $\mathrm{W}$ atom in between. Ohara et al. [8] have experimentally show that the transition metal-silicon binary clusters anions $\mathrm{MSi}_{n}^{-}(\mathrm{M}=\mathrm{Ti}, \mathrm{Hf}, \mathrm{Mo}$ and $\mathrm{W})$ were produced by a double-laser vaporization method. Lu and Nagase [21], Hagelberg et al. [22] and Sen and Mitas [23] have studied various kinds of metal atoms for doping Si clusters. Guo et al. [24] systematically investigated the geometry, stability, electronic and magnetic properties of transition metal encapsulated $\mathrm{MSi}_{n}(\mathrm{M}=\mathrm{Sc}$ to $\mathrm{Zn} ; n=8-16)$ clusters. They found that the transition metal atom would fall into the center of the $\mathrm{Si}$ outer frame, forming a metal-encapsulated Si cage. The stable cluster $\mathrm{C} 60$ has been produced by laser irradiation of graphite [25]. Beck experimentally detected the clusters of $\mathrm{MSi}_{n}$ (Cr, Mo, and W) particularly for the sizes of $n=15$ and $n=16[26,27]$. An experimental evidence of high stability of $\mathrm{M}_{\mathrm{Si}} \mathrm{i}_{16}(\mathrm{M}=\mathrm{Sc}, \mathrm{Ti}$, and $\mathrm{V})$ clusters was established using mass spectrometry and anion photoelectron spectroscopy by Koyasu et al. [28] and the exceptional stability of $\mathrm{MSi}_{16}(\mathrm{M}=\mathrm{Ti}, \mathrm{Zr}$, and $\mathrm{Hf})$ has been experimentally confirmed by Furuse et al. [29].

Recent experimental and theoretical works show that the very stable structures like $\mathrm{M} @ \mathrm{Si}_{16}$ clusters $\left(\mathrm{M}=\mathrm{Sc}^{-}\right.$, $\mathrm{Ti}$, or $\mathrm{V}^{+}$) have interesting properties which suggests the use of these clusters as basic units in the assembly of optoelectronic materials which are an original class of nanostructured solids with specific structures and properties [30-32]. Reis and Pacheco [33] show that bulk materials assembled with $\mathrm{MSi}_{16}(\mathrm{M}=\mathrm{Ti}, \mathrm{Zr}$, and $\mathrm{Hf})$, when appropriately doped, may exhibit high-temperature superconducting properties. These clusters of silicon doped with iron can find technological applications promising, for example, for the magnetic information storage and could be useful for spintronics and other magnetic device 
applications [34]. Also, the doped silicon clusters could be used to tailor band gaps with heretofore unattainable specificity. This could lead to applications in both the microelectronics and optoelectronic industries.

In this paper, we present a theoretical investigation of the properties of $\mathrm{Fe}$ atoms doped silicon cage clusters, $\mathrm{Si}_{n-m} \mathrm{Fe}_{m}$ with different sizes $(19 \leq n \leq 24)$ and $(1 \leq m \leq 3)$, by using density functional theory (DFT) calculation with generalized gradient approximation (GGA) implemented in SIESTA packages [35]. The most of the previous works study the silicon structures with low sizes $(<16$ atoms) doped with one atom of transition metal. The question is what about the bigger structures $(n>16)$ when they are doped by more of one metal transition atom $(\mathrm{Fe})$ ? Structural, electronic and magnetic properties of these clusters were studied as a function of the number and the positions of Fe atoms in the structure. After introduction and brief review of computational methodology, we proceed with the presentation of the results and their discussion. Finally, we end our main study by a conclusion.

\section{Computational details}

To perform our calculation we have used the SIESTA simulation packages [35]. This method employs linear combination of pseudo atomic orbital as basis set. Our calculations were done within DFT [36, 37] in the GGA. Our pseudopotentials were generated according to the Troullier-Martins scheme with taking into the count the effect of core electrons [38]. The GGA of the density functional has been used with the exchange-correlation energy functional parameterized by Perdew and Zunger [39] and Perdew, Burke, and Ernzernhof (PBE) [40]. We use double $\zeta(\mathrm{DZ})$ bases with polarization function for $\mathrm{Fe}$ and single $\zeta(\mathrm{SZ})$ for Si. The choice of orbital SZ for the silicon was optimized and verified in test calculation that it does not affect the results of calculation. However, the choice of the DZ for Fe is very necessary. In order to avoid interactions between clusters, our system is modelled by a big cubic supercell with 40 A edge including enough vacuum around the cluster in neighbouring cells sufficiently large. During simulation volume of the system was kept constant. Self-consistent field calculations are carried out with convergence criterion of $10^{-3}$ a.u. on the total energy and electron density. The $\Gamma$-point approximation was used for the Brillouin zone sampling.

In structural optimizations, using a conjugate gradient method, and in dynamical simulation, the HellmannFeynman force on the atoms is evaluated between each optimization or molecular dynamics step. The clusters were allowed to relax until the interatomic forces were smaller than $10^{-2} \mathrm{eV} / \AA$. To obtain the electronic structure of the system the standard diagonalisation solution of the Hamiltonian is used. For the systems with number of atoms less than 100 atoms, the diagonalisation method is competitive and more convenient than the order- $N$ method. The binding energies reported be- low were calculated by subtracting the sum of the energies for the individual atoms (computed using the same method and basis sets as in the cluster calculations) from the cluster energy. The used MeshCutoff is $150 \mathrm{Ry}$ and PAO.EnergyShift of $50 \mathrm{meV}$. These values are adequate and not affect significantly the results of calculations.

\section{Results and discussions}

\subsection{Structure of $S i_{n-m} F e_{m}$ clusters}

The lowest energy structures and low-lying isomers obtained for the $\mathrm{Si}_{n-m} \mathrm{Fe}_{m}$ clusters with different sizes $(19 \leq n \leq 24),(1 \leq m \leq 3)$ are shown in Figs. 1-6. The lowest energy structures of pure silicon are those obtained in our previous work [41, 42]. They are reported here in Table for comparison. The most stable isomers $\mathrm{Si}_{n-m} \mathrm{Fe}_{m}$ are indicated in bold character in Table. The optimizations have been performed without any symmetry constraint.
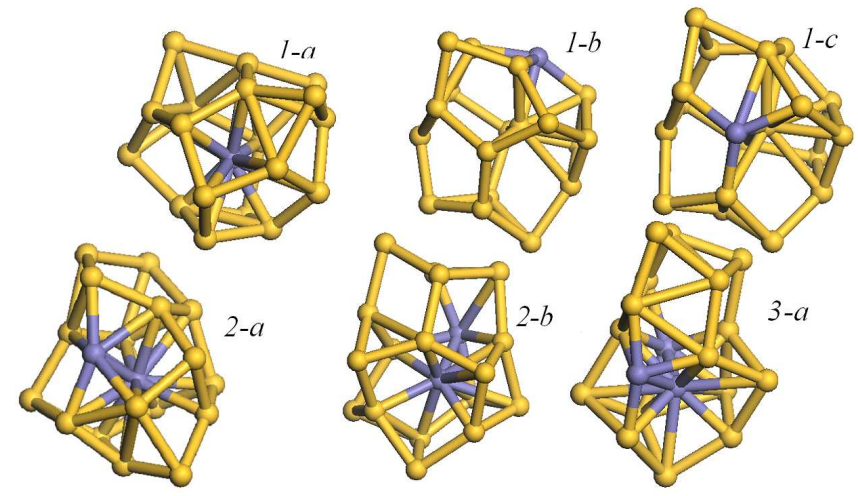

Fig. 1. Ground-state geometries of $\mathrm{Si}_{18}-\mathrm{Fe}(1-a, 1-b$ and 1-c), $\mathrm{Si}_{17}-\mathrm{Fe}_{2}$ (2-a and 2-b) and $\mathrm{Si}_{16}-\mathrm{Fe}_{3}$ (3-a) clusters.

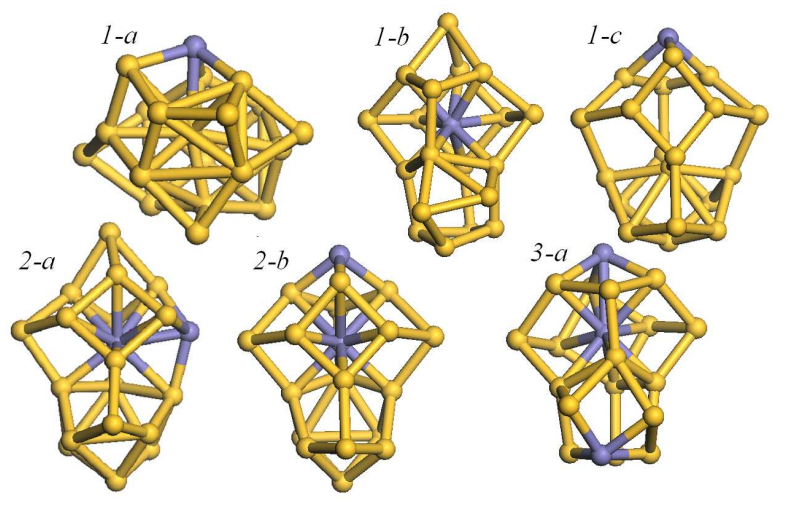

Fig. 2. Ground-state geometries of $\mathrm{Si}_{19}-\mathrm{Fe}$ (1- $a, 1-b$ and 1-c), $\mathrm{Si}_{18}-\mathrm{Fe}_{2}(2-a$ and $2-b)$ and $\mathrm{Si}_{17}-\mathrm{Fe}_{3}(3-a)$ clusters.

To determine the effect of Fe atoms, we study several initial structures, as a function of the number and the 

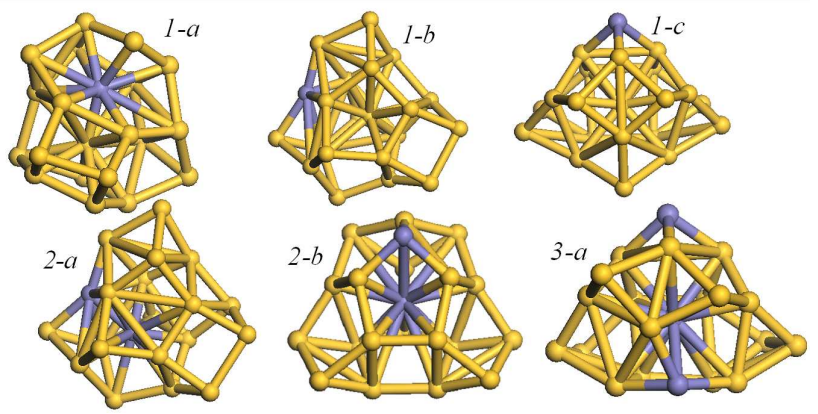

Fig. 3. Ground-state geometries of $\mathrm{Si}_{20}-\mathrm{Fe}$ (1- $a, 1-b$ and 1-c), $\mathrm{Si}_{19}-\mathrm{Fe}_{2}$ (2- $a$ and $\left.2-b\right)$ and $\mathrm{Si}_{18}-\mathrm{Fe}_{3}$ (3-a) clusters.
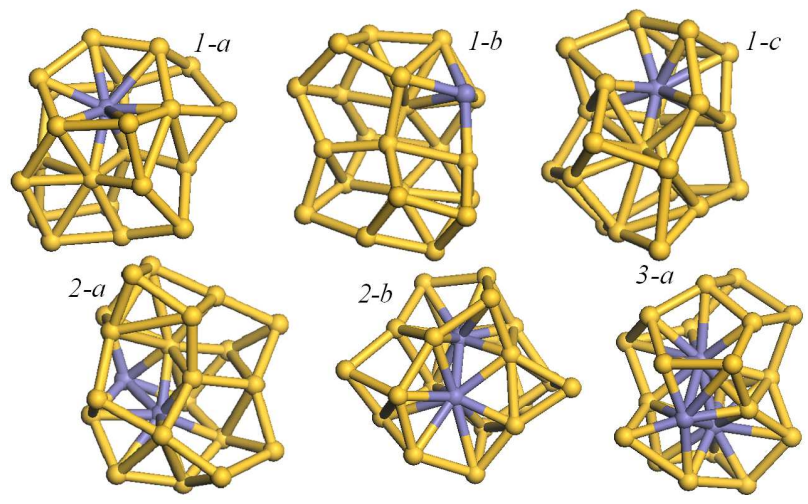

Fig. 4. Ground-state geometries of $\mathrm{Si}_{21}-\mathrm{Fe}$ (1- $a, 1-b$ and $1-c), \mathrm{Si}_{20}-\mathrm{Fe}_{2}$ (2-a and 2-b) and $\mathrm{Si}_{19}-\mathrm{Fe}_{3}(3-a)$ clusters.

positions of Fe atoms in pure silicon clusters for each size, and proceed to perform structural optimizations using total energy calculations. Here we report only the most stable ones. We first note that in all these clusters Fe occupies a substitutional site and bonds with $\mathrm{Si}$ atoms. As we can see in Figs. 1-6 and Table, the geometric,
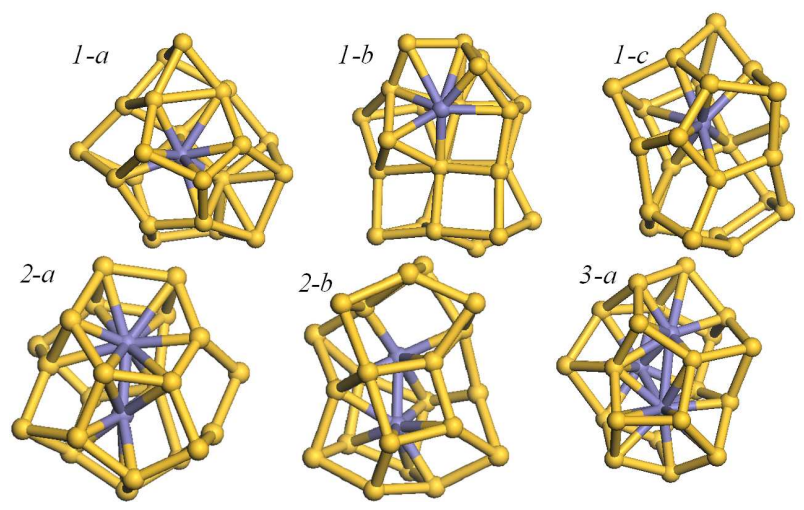

Fig. 5. Ground-state geometries of $\mathrm{Si}_{22}-\mathrm{Fe}(1-a, 1-b$ and $1-c), \mathrm{Si}_{21}-\mathrm{Fe}_{2}(2-a$ and $2-b)$ and $\mathrm{Si}_{20}-\mathrm{Fe}_{3}(3-a)$ clusters.

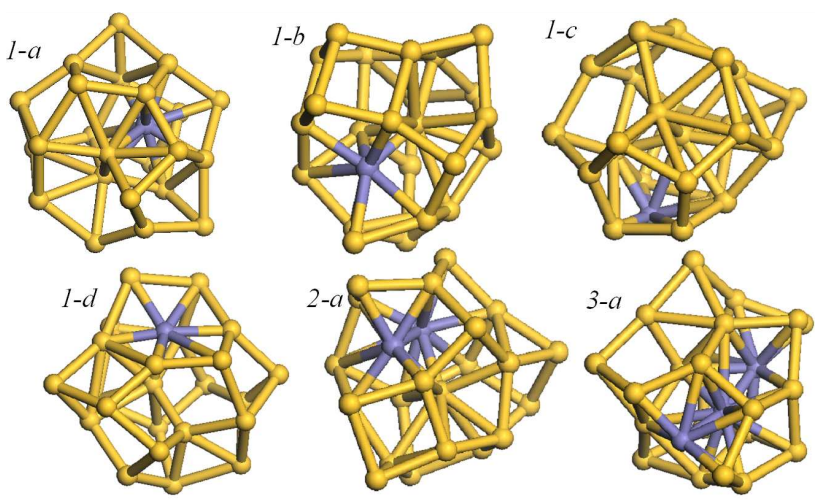

Fig. 6. Ground-state geometries of $\mathrm{Si}_{23}-\mathrm{Fe}(1-a, 1-b$, 1-c and 1-d), $\mathrm{Si}_{22}-\mathrm{Fe}_{2}(2-a)$ and $\mathrm{Si}_{21}-\mathrm{Fe}_{3}(3-a)$ clusters.

the electronic and the magnetic properties of the clusters depend largely on the position and on the number of Fe atoms. In the following we describe the geometric structure of our clusters.

In the case of $n=19$ atoms: the ground state structure is (1-a) with one doping atom localised at the center unlike in the other case (1-b) and 1-c) in which the doped atom is localised at the surface of the cluster. The structure (2-b) and (2-c) with two doping Fe atoms, the first one at the center and second at the surface, have systematically similar binding energy. The structure (3- $a$ ) with three doping $\mathrm{Fe}$ atoms is the least stable but with highest magnetic moment of $7.99 \mu_{\mathrm{B}}$.

In the case of $n=20$ atoms: the ground state structure is (1-b) with one doping atom localised at the center unlike in the other case $(1-a)$ and (1-c). This result proves that the cluster is more stable when the doping atom is located at the center of the structure. This result situation can be clearly observed in all other cases up to 24 atoms as we can see in Table and shown in Figs. 1-6.

In the case of $n=21$ atoms: we obtained three structure isomers with one doping Fe atom almost with the same binding energy per atom. The structure (1-c) is close to $C_{s}$ symmetry. In the case of two doping Fe atoms, we obtained two structures with near $C_{s}$ symMetry. The structure $(2-b)$ has the smallest HOMO-LUMO gap. The structure (3- $a$ ) with three doping Fe atoms has a similar geometry to that of $(1-c)$ structure but with the smallest binding energy per atom.

In the case of $n=22$ atoms: the different obtained structures are generally with elongated shape and become more compact when the number of doping Fe atoms increases. For the three types of structures $(m=1,2$ and 3 ), the most stable is generally the one which has the doping Fe atoms in the core of the cluster as we can see in Fig. 4 and Table. The best structure is (1-c) with binding energy of $5.071 \mathrm{eV} /$ atom. 
TABLE

Point group, binding energy per atom $E_{\mathrm{b}}(\mathrm{eV} /$ atom) and HOMO-LUMO gap in spin up $\Delta E(\mathrm{eV}) \uparrow$ and spin down $\Delta E(\mathrm{eV}) \downarrow$ and total magnetic moments $\mu\left(\mu_{\mathrm{B}}\right)$ of $\mathrm{Si}_{n-m} \mathrm{Fe}_{m}$ clusters.

\begin{tabular}{|c|c|c|c|c|c|c|c|c|c|}
\hline Cluster size $(n)$ & & $\mathrm{Si}_{\mathrm{n}}$ & & & & & $\mathrm{i}_{n-m} \mathrm{Fe}_{m}$ & & \\
\hline \multirow{7}{*}{19} & Group & $E_{\mathrm{b}}[\mathrm{eV} /$ atom $]$ & $\Delta E[\mathrm{eV}]$ & $m$ & Group & $E_{\mathrm{b}}[\mathrm{eV}]$ & $\Delta E[\mathrm{eV}] \uparrow$ & $\Delta E[\mathrm{eV}] \downarrow$ & $\mu\left[\mu_{\mathrm{B}}\right]$ \\
\hline & \multirow{6}{*}{$C_{1}$} & \multirow{6}{*}{4.6785} & \multirow{6}{*}{0.6472} & 1- $a$ & $C_{1}$ & 4.995 & 0.740 & 0.539 & 3.99 \\
\hline & & & & $1-b$ & $C_{1}$ & 4.947 & 0.756 & 0.384 & 3.99 \\
\hline & & & & $1-c$ & $C_{1}$ & 4.954 & 0.607 & 0.536 & 3.99 \\
\hline & & & & $2-a$ & $C_{1}$ & 4.665 & 0.598 & 0.274 & 6.00 \\
\hline & & & & $2-b$ & $\boldsymbol{C}_{1}$ & 4.686 & 0.772 & 0.390 & 5.99 \\
\hline & & & & 3- $a$ & $C_{1}$ & 4.338 & 0.479 & 0.423 & 7.99 \\
\hline \multirow{6}{*}{20} & \multirow{6}{*}{$C_{s}$} & \multirow{6}{*}{4.6789} & \multirow{6}{*}{0.9186} & 1- $a$ & $C_{1}$ & 4.964 & 0.676 & 0.461 & 3.99 \\
\hline & & & & $1-b$ & $\boldsymbol{C}_{1}$ & 5.059 & 0.534 & 0.632 & 1.99 \\
\hline & & & & $1-c$ & $C_{s}$ & 4.954 & 0.804 & 0.607 & 3.99 \\
\hline & & & & $2-a$ & $C_{1}$ & 4.685 & 0.421 & 0.488 & 5.99 \\
\hline & & & & $2-b$ & $C_{1}$ & 4.689 & 0.672 & 0.429 & 4.00 \\
\hline & & & & $3-a$ & $C_{1}$ & 4.354 & 0.559 & 0.596 & 7.99 \\
\hline \multirow{6}{*}{21} & \multirow{6}{*}{$C_{s}$} & \multirow{6}{*}{4.434} & \multirow{6}{*}{0.961} & $1-a$ & $\boldsymbol{C}_{1}$ & 5.063 & 0.620 & 0.390 & 1.99 \\
\hline & & & & $1-b$ & $C_{1}$ & 5.022 & 0.863 & 0.360 & 3.99 \\
\hline & & & & $1-c$ & $C_{s}$ & 5.008 & 0.861 & 0.495 & 3.99 \\
\hline & & & & $2-a$ & $C_{1}$ & 4.763 & 0.657 & 0.368 & 5.99 \\
\hline & & & & $2-b$ & $C_{1}$ & 4.710 & 0.694 & 0.237 & 7.99 \\
\hline & & & & $3-a$ & $C_{1}$ & 4.362 & 0.744 & 0.472 & 11.96 \\
\hline \multirow{6}{*}{22} & \multirow{6}{*}{$C_{1}$} & \multirow{6}{*}{4.389} & \multirow{6}{*}{0.917} & 1- $a$ & $C_{1}$ & 5.021 & 0.601 & 0.427 & 3.99 \\
\hline & & & & $1-b$ & $C_{1}$ & 4.999 & 0.761 & 0.266 & 3.99 \\
\hline & & & & $1-c$ & $C_{1}$ & 5.071 & 0.681 & 0.518 & 1.99 \\
\hline & & & & $2-a$ & $C_{1}$ & 4.729 & 0.666 & 0.509 & 7.95 \\
\hline & & & & $2-b$ & $C_{1}$ & 4.813 & 0.498 & 0.481 & 4.00 \\
\hline & & & & 3-a & $C_{1}$ & 4.544 & 0.455 & 0.522 & 5.99 \\
\hline \multirow{6}{*}{23} & \multirow{6}{*}{$C_{1}$} & \multirow{6}{*}{4.354} & \multirow{6}{*}{0.809} & 1- $a$ & $C_{1}$ & 5.122 & 0.695 & 0.535 & 2.00 \\
\hline & & & & $1-b$ & $C_{1}$ & 5.042 & 0.633 & 0.395 & 2.00 \\
\hline & & & & $1-c$ & $C_{1}$ & 5.087 & 0.695 & 0.677 & 2.00 \\
\hline & & & & $2-a$ & $C_{1}$ & 4.860 & 0.710 & 0.505 & 4.00 \\
\hline & & & & $2-b$ & $C_{1}$ & 4.823 & 0.473 & 0.615 & 4.00 \\
\hline & & & & $3-a$ & $C_{1}$ & 4.629 & 0.498 & 0.455 & 5.99 \\
\hline \multirow{6}{*}{24} & \multirow{6}{*}{$C_{1}$} & \multirow{6}{*}{4.425} & \multirow{6}{*}{1.041} & 1- $a$ & $C_{1}$ & 5.107 & 0.635 & 0.683 & 2.00 \\
\hline & & & & $1-b$ & $C_{1}$ & 5.102 & 0.628 & 0.428 & 2.00 \\
\hline & & & & $1-c$ & $C_{1}$ & 5.075 & 0.593 & 0.476 & 3.99 \\
\hline & & & & $1-d$ & $C_{1}$ & 5.080 & 0.550 & 0.622 & 3.95 \\
\hline & & & & $2-a$ & $C_{1}$ & 4.854 & 0.528 & 0.521 & 5.99 \\
\hline & & & & 3- $a$ & $C_{1}$ & 4.572 & 0.337 & 0.388 & 7.87 \\
\hline
\end{tabular}

In the case of $n=23$ atoms: we have also observed different structures with elongated shape as in the case of 22 atoms. The best one is (1- $a$ ) with binding energy of $5.122 \mathrm{eV} /$ atom and one doping Fe atom at the center of the cluster.

In the case of $n=24$ atoms: all obtained structures are in like-spherical shape and very compact. The best one is (1-a) with binding energy of $5.107 \mathrm{eV} /$ atom and one doping Fe atom at the center of structure.

\subsection{Relative stability and electronic properties of $S i_{n-m} F e_{m}$ clusters}

We now study the relative stability of $\mathrm{Si}_{n-m} \mathrm{Fe}_{m}$ clusters by the binding energies per atom $\left(E_{\mathrm{b} / \text { atom }}\right)$. To obtain the binding energies for the lowest energy structures of the $\mathrm{Si}_{n-m} \mathrm{Fe}_{m}$ clusters we have used the following formula:

$$
\begin{aligned}
& E_{\mathrm{b} / \text { atom }}\left(\mathrm{Si}_{n-m} \mathrm{Fe}_{m}\right)=[(n-m) E(\mathrm{Si})+m E(\mathrm{Fe}) \\
& \left.\quad-E\left(\mathrm{Si}_{n-m} \mathrm{Fe}_{m}\right)\right] / n,
\end{aligned}
$$


where $E(\mathrm{Si})$ is the total energy of the free $\mathrm{Si}$ atom, $E(\mathrm{Fe})$ is the total energy of free Fe atom and $E\left(\mathrm{Si}_{n-m} \mathrm{Fe}_{m}\right)$ is the total calculated energy of the cluster.

It is interesting to check if the doped $\mathrm{Si}_{n}$ clusters could be stabilized by the introduction of the transition metal Fe atoms. This can be simply concluded by comparing the binding energy of doped $\mathrm{Si}_{n-m} \mathrm{Fe}_{m}$ clusters with that of pure silicon of the same size.

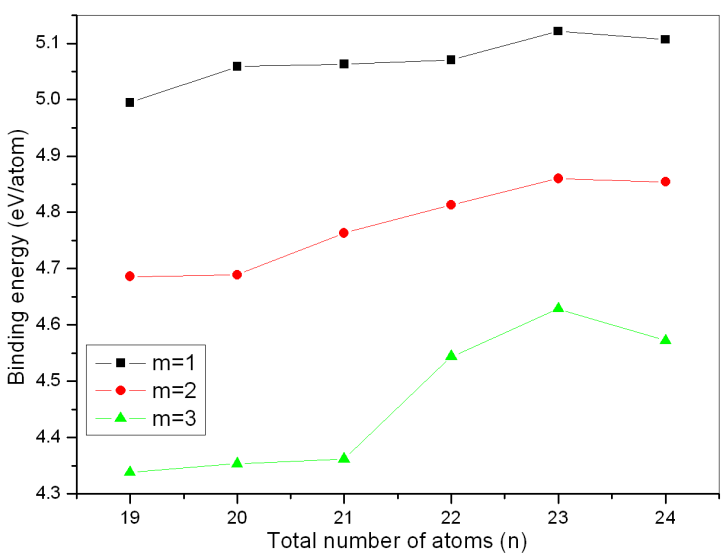

Fig. 7. Binding energy per atom of $\mathrm{Si}_{n-m} \mathrm{Fe}_{m}$ clusters.

The obtained cohesive energies per atom for each cluster are reported in Table and shown in Fig. 7 for the most stable structures. For comparison, the averaged binding energies of pure silicon clusters are also reported in Table. The doping with one atom enhances the binding energy of the clusters, which implies that the doping of the $\mathrm{Fe}$ atom improves the stability of pure $\mathrm{Si}_{n}$ clusters. This is due to the higher bond strength of $\mathrm{Fe}-\mathrm{Si}$ than that of $\mathrm{Si}-\mathrm{Si}$.

As we can see in Table, the binding energies of the doped silicon clusters with one Fe atom are larger than the corresponding pure silicon clusters in all clusters sizes studied here. This suggests that the impurity Fe atom increases the stability of the doped silicon clusters, which is caused by the improvement of some bond strength. This improvement in the stability of the silicon structure cage is also observed, with a less degree, in the case of two doping Fe atoms. This stabilization is mainly due to the charge transfer between Fe atoms to the Si atoms [3].

The cohesive energies of the best isomers increase as the cluster size increases for the different number of doped Fe atoms ( $m=1,2$ and 3 ) which means that these clusters can continue to gain energy during the growth process. Local peaks of binding energy per atom are found at $n=20$ and 23 for $m=1$ and at $n=23$ for $m=1,2$ and 3 implying that these clusters are more stable than their neighbouring clusters.

Also, as we can see clearly in Table and Fig. 7, the cohesive energies of clusters with one $\mathrm{Fe}$ atom are very important when compared to those having two Fe atoms which are also important comparatively to those having three Fe atoms. This evolution seems to be in very good agreement with the hypothesis that when the number of Fe atoms replacing silicon atoms becomes increasingly important and leads to obtain a cluster of iron instead of silicon. Consequently, the increase of the size leads to a cohesive energy per atoms close to that of bulk iron which is experimentally measured to be $4.28 \mathrm{eV} /$ atom [43].

The energy difference between the highest occupied molecular orbital (HOMO) and lowest unoccupied molecular orbital (LUMO) is considered to be a good indicator of the electronic stability for the intermediate size clusters. The HOMO-LUMO gap energies in spin up and spin down of the different clusters are given in Table. It is interesting to note that the HOMO-LUMO gaps in spin up and spin down of the $\mathrm{Si}_{n-m} \mathrm{Fe}_{m}$ clusters are slightly smaller than the corresponding calculated pure silicon clusters, indicating that the chemical activity of $\mathrm{Si}_{n-m} \mathrm{Fe}_{m}$ is higher than those of the pure silicon clusters, and that the inserted Fe atoms raise the chemical activity and increase the metallic character of $\mathrm{Si}_{n-m} \mathrm{Fe}_{m}$ clusters. Also, we observe than the clusters have large HOMO-LUMO gap for spin up electrons while spin down electrons have a very small HOMO-LUMO gap. This trend will have an interesting repercussion on electronic conductance through these clusters which could be considered as interesting properties in spin electronics applications for example as spin filter. The nature of the chemical bond can be understood by analyzing the electron density of states distribution (DOS). In our case, we calculated the difference between the electronic densities of states of the doped silicon clusters.

The results for the most stable structure in each cluster size are shown in Fig. 8 for one, two and three doping Fe atoms. The positive bottom (spin up) and negative top parts (spin down) of the electronic density are plotted separately for a convenient analysis. As can be seen from total DOS curves, all of structures show metallic character due to the the absence of a gap around the Fermi level in all case studied here. Also, DOS curves reveal that the very intense peak shifts slightly to lower energy at the Fermi level when the number of Fe atoms increase in each cluster size. This effect can be explained by the hybridization phenomenon between $\mathrm{Si}$ and Fe atoms. This hybridization becomes very important as the radius between Fe and neighbouring Si atoms decreases.

\subsection{Magnetic properties of $S i_{n-m} F e_{m}$ clusters}

Based on the optimized geometries, the magnetic properties of $\mathrm{Si}_{n-m} \mathrm{Fe}_{m}$ clusters have been computed and the results are shown in Table. We observe that the doping iron atoms have much effect on the magnetism for the pure silicon clusters. The silicon clusters which are not magnetic in their pure state become magnetic after the substitution of Fe atoms in the structures. These magnetic properties change with the number of Fe atoms in the clusters, as we can see in Table: the magnetic moment increases with increase of the number of Fe atoms in the cluster.

The magnetic character observed in our clusters is due to the charge transfer between $d$ atomic orbitals of Fe 


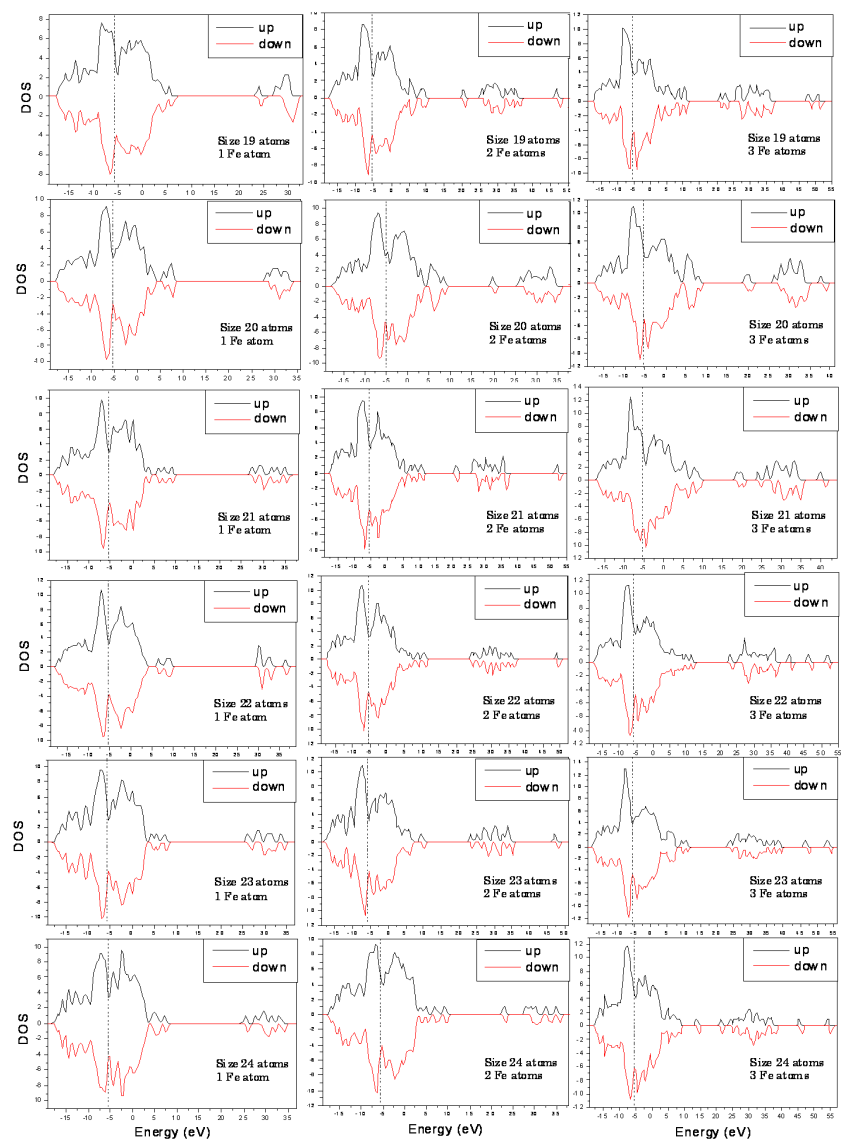

Fig. 8. Total density of states of $\mathrm{Si}_{n-m} \mathrm{Fe}_{m}(19 \leq n \leq$ $24,1 \leq m \leq 3)$ clusters. The vertical dashed line indicates the position of the Fermi level in each DOS curve.

atoms and the $s$ atomic orbitals of Si atoms which implies that Fe atoms act as electron donors [3]. We also observe that the total magnetic moment of $\mathrm{Si}_{n-m} \mathrm{Fe}_{m}$ is not quenched when the doping $\mathrm{Fe}$ atoms are located at the surface of the clusters: it is almost equal to $4 \mu_{\mathrm{B}}$ in the case of one doping Fe atom, $8 \mu_{\mathrm{B}}$ in the case of two doping Fe atoms and $12 \mu_{\mathrm{B}}$ for three doping $\mathrm{Fe}$ atoms. However, the magnetic moment becomes much quenched when the doping Fe atoms are located at the center of the structures as in the case of following structures: $n=19$ $(2-b)$ and $(3-a) ; n=20(1-b),(2-a)$ and $(3-a) ; n=21$ (1-a), (2-a) and (2-b); $n=22(1-c),(2-b)$ and (3-a); all cases of $n=23$ atoms; $n=24$ (1-a), (1-b), (2-a) and (3-a). In the case of structures with one Fe atom, the coupling between the $\mathrm{Fe}$ atom and the $\mathrm{Si}$ cluster is so weak that the Fe atom is almost an isolated atom when this Fe atom is located at the surface of structure.

\section{Conclusion}

We have systematically studied the effect of iron atoms on the properties of silicon cage clusters in the range of sizes from 19 to 24 atoms. Geometric structures, stabilities, electronic, and magnetic properties of these clusters have been investigated by using density functional methods. The most stable structures have been identified and analysed. We have obtained different isomer structures with different properties for each cluster size. The substitution of iron atoms in silicon clusters leads to more stable silicon cage structures especially those doped with one then with two iron atoms. The HOMO-LUMO gaps in spin up and spin down of $\mathrm{Si}_{n-m} \mathrm{Fe}_{m}$ clusters are slightly smaller than in the corresponding pure silicon clusters and the HOMO-LUMO gaps for spin up are generally larger than those of spin down. These results represent an interesting property for potential spin-electronic applications. In all cases $(m=1,2$ or 3$)$, the binding energy shows a smooth variation and increases with the increase of cluster size. The silicon clusters which are not magnetic in their pure state become magnetic after the substitution of iron atoms and the magnetic moment increases with increase of the number of iron atoms in the cluster.

\section{References}

[1] V. Kumar, Comp. Mat. Sci. 30, 260 (2004).

[2] J. Wang, Q. Min Ma, R. Ping Xu, Y. Liu, Y. Cheng Li, Phys. Lett. A 373, 2869 (2009).

[3] S. Mahtout, M.A. Belkhir, Phys. Lett. A 360, 384 (2006).

[4] D. Hossain, C.U. Pittman Jr., S.R. Gwaltney, Chem. Phys. Lett. 451, 93 (2008).

[5] M. Ohara, K. Koyasu, A. Nakajima, K. Kaya, Chem. Phys. Lett. 371, 490 (2003).

[6] A.P. Yang, Z.Y. Ren, P. Guo, G.H. Wang, J. Mol. Struct. 856, 88 (2008).

[7] W. Su, P. Qian, Y. Liu, J. Shen, N.X. Chen, Comp. Phys. Commun. 181, 726 (2010).

[8] A. Gao, G. Li, Y. Chang, H. Chen, D. Finlow, Q. Li, Inorg. Chim. Acta 367, 51 (2011).

[9] A. Gao, G. Li, Y. Chang, H. Chen, Q. Li, J. Mol. Struct. 961, 88 (2010).

[10] B. Li, G. Wang, M. Ye, G. Yang, C. Yao, J. Mol. Struct. 820, 128 (2007).

[11] J. Wang, J. Zhao, L. Ma, B. Wang, G. Wang, Phys. Lett. A 367, 335 (2007).

[12] M. Menon, K.R. Subbaswamy, Chem. Phys. Lett. 219, 219 (1994).

[13] M. Broyer, M. Pellarin, B. Baguenard, J. Lormé, J.L. Vialle, P. Melinon, J. Tuaillon, V. Dupuis, B. Prevel, A. Perez, Mater. Sci. Forum 232, 27 (1996).

[14] H. Hiura, T. Miyazaki, T. Kanayama, Phys. Rev. Lett. 86, 1733 (2001).

[15] T. Miyazaki, H. Hiura, T. Kanayama, Phys. Rev. B 66, 121403 (2002).

[16] G. Mpourmpakis, G.E. Froudakis, Phys. Rev. B 68, 125407 (2003).

[17] V. Kumar, Y. Kawazoe, Phys. Rev. Lett. 87, 045503 (2001); V. Kumar, Y. Kawazoe, Phys. Rev. B 65, 073404 (2002). 
[18] M. Menon, A.N. Andriotis, G. Froudakis, Nanoletters 2, 301 (2002).

[19] A.N. Andriotis, G. Mpourmpakis, G. Froudakis, M. Menon, New J. Phys. 4, 78 (2002).

[20] V. Kumar, Y. Kawazoe, Phys. Rev. Lett. 88, 235504 (2002).

[21] J. Lu, S. Nagase, Phys. Rev. Lett. 90, 115506 (2003).

[22] F. Hagelberg, C. Xiao, W.A. Lester Jr., Phys. Rev. B 67, 035426 (2003).

[23] P. Sen, L. Mitas, Phys. Rev. B 68, 155404 (2003).

[24] L.J. Guo, G.F. Zhao, Y.Z. Gu, X. Liu, Z. Zeng, Phys. Rev. B 77, 195417 (2008).

[25] H.W. Kroto, J.R. Heath, S.C. O'Brien, R.F. Curl, R.E. Smalley, Nature 318, 162 (1985).

[26] S.M. Beck, J. Chem. Phys. 87, 4233 (1987).

[27] S.M. Beck, J. Chem. Phys. 90, 6306 (1989).

[28] K. Koyasu, M. Akutsu, M. Mitsui, A. Nakajima, J. Am. Chem. Soc. 127, 4998 (2005).

[29] S. Furuse, K. Koyasu, J. Atobe, A. Nakajima, J. Chem. Phys. 129, 064311 (2008).

[30] M.B. Torres, E.M. Fernaández, L.C. Balbaás, J. Phys. Chem. C 115, 335 (2010).

[31] M.B. Torres, E.M. Fernández, L.C. Balbás, Int. J. Quant. Chem. 111, 444 (2010).

[32] C.L. Reis, J.L. Martins, J.M. Pacheco, Phys. Rev. B 76, 233406 (2007).
[33] C.L. Reis, J.M. Pacheco, Phys. Rev. B 82, 155440 (2010).

[34] B.E. Kane, Nature 393, 133 (1998).

[35] P. Ordejón, E. Artacho, J.M. Soler, Phys. Rev. B (Rapid Comm.) 53, R10441 (1996); J.M. Soler, E. Artacho, J.D. Gale, A. García, J. Junquera, P. Ordejón, D. Sánchez-Portal, J. Phys., Condens. Matter. 14, 2745 (2002).

[36] P. Hohenberg, W. Kohn, Phys. Rev. B 136, 864 (1964).

[37] W. Kohn, L.J. Sham, Phys. Rev. 140, A1133 (1965).

[38] N. Troullier, J.L. Martins, Phys. Rev. B 43, 1993 (1991).

[39] J.P. Perdew, A. Zunger, Phys. Rev. B 23, 5048 (1981).

[40] J.P. Perdew, K. Burke, M. Ernzernhof, Phys. Rev. Lett. 77, 3865 (1996).

[41] M.A. Belkhir, S. Mahtout, I. Belabbas, M. Samah, Physica E 31, 86 (2006).

[42] S. Mahtout, M.A. Belkhir, Physica E 40, 2884 (2008).

[43] P.H.T. Philipsen, E.J. Baerends, Phys. Rev. B 54, 5326 (1996). 